\title{
Identification of novel sulfur-containing bacterial polyesters: biosynthesis of poly(3-hydroxy-S-propyl- $\omega$-thioalkanoates) containing thioether linkages in the side chains
}

\author{
Christian Ewering, ${ }^{1}$ Tina Lütke-Eversloh, ${ }^{1}$ Heinrich Luftmann ${ }^{2}$ \\ and Alexander Steinbüchel ${ }^{1}$
}
1 Institut für Mikrobiologie, Westfälische Wilhelms- Universität Münster, Corrensstraße 3, D-48149 Münster, Germany
2 Institut für Organische Chemie, Westfälische Wilhelms-Universität Münster, Corrensstraße 40, D-48149 Münster, Germany

\begin{abstract}
Author for correspondence: Alexander Steinbüchel. Tel: +49 251 8339821. Fax: +492518338388. e-mail: steinbu@uni-muenster.de
\end{abstract}

This study describes the biosynthesis of novel sulfur-containing polyhydroxyalkanoates (PHAs), which consist exclusively of hydroxypropylthioalkanoic acid containing thioether groups in the side chains. In addition, the utilization of alkylthioalkanoic acids ( = thia fatty acids) by various bacteria was investigated. Based on feedings with propylthiooctanoic acid (PTO) or propylthiohexanoic acid, the metabolically engineered PHAnegative mutant PHB-4 of Ralstonia eutropha, which harbours plasmid pBBR1:: phaC1 expressing the PHA synthase of Pseudomonas mendocina, synthesized two novel poly(3-hydroxy-S-propyl- $\omega$-thioalkanoic) acids [poly(3HPTA)s]. A terpolyester consisting of 3-hydroxypropylthiobutyric acid (3HPTB), 3-hydroxypropylthiohexanoic acid (3HPTHx) and 3-hydroxypropylthiooctanoic acid (3HPTO) was synthesized from PTO, whereas a co-polyester of 3HPTB and 3HPTHx was synthesized from propylthiohexanoic acid. Fed-batch fermentation of $R$. eutropha PHB-4(pBBR1::phaC1) on PTO was done on a 26-litre scale, providing a cell density of $7 \cdot 3 \mathrm{~g} \mathrm{I}^{-1}$, from which $45 \mathrm{~g}$ of the novel poly(3HPTB-co-3HPTHx-co-3HPTO) were isolated. The chemical structures of the poly(3HPTA)s were identified by gas chromatography/mass spectrometry, elemental sulfur analysis, partial pyrolysis and detailed mass spectrometric analysis, exhibiting 3HPTB, 3HPTHx and 3HPTO as constituents. These novel, hitherto undescribed, constituents of PHAs were randomly distributed in the co-polyesters.

Keywords: polyhydroxyalkanoates, Ralstonia eutropha, metabolic engineering, thia fatty acids, alkylthioalkanoate

\section{INTRODUCTION}

Polyhydroxyalkanoates (PHAs) represent one of eight classes of biopolymers, and they occur abundantly as storage compounds for energy and carbon in a large variety of bacteria and archaea (Anderson \& Dawes, 1990; Hocking \& Marchessault, 1994). They are synthe-

Abbreviations: ATA, alkylthioalkanoic acid; BTV, butylthiovaleric acid GC/MS, gas chromatography and mass spectrometry; 3HD, 3-hydroxydecanoic acid; 3HPTB, 3-hydroxypropylthiobutyric acid; 3HPTHx, 3-hydroxypropylthiohexanoic acid; 3HPTO, 3-hydroxypropylthiooctanoic acid; PHA, polyhydroxyalkanoate; $\mathrm{PHA}_{\mathrm{MCL}}$, medium-chain-length polyhydroxyalkanoate; poly(3HPTA), poly(3-hydroxy-S-propyl- $\omega$-thioalkanoate); PTO, propylthiooctanoic acid; PTUD, propylthioundecanoic acid. sized by PHA synthases, which exhibit a rather low substrate specificity and use various hydroxyalkanoylCoA thioesters as their substrates. Due to this wide substrate range, a variety of different PHAs can be synthesized, and approximately 150 different hydroxyalkanoates have been identified as constituents (Steinbüchel \& Valentin, 1995). These constituents include hydroxyalkanoic acids of chain lengths ranging from 3 to 16 carbon atoms with the hydroxyl group positioned at the carbon atoms numbered $2,3,4,5$ or 6 , with one or even two additional methyl groups in that region of the hydroxyalkanoic acid representing the polyester backbone and also 3-hydroxyalkanoic acids with alkyl side chains containing various functional groups (Steinbüchel \& Valentin, 1995, and references cited therein). 
Due to the interest of industry in novel biodegradable polymeric materials, and due to academic questions, PHA synthases and PHA synthesis pathways have been investigated in great detail in the past. PHA synthases from more than 50 different bacteria have been cloned, and several of them have been thoroughly analysed at a biochemical and molecular level (Rehm \& Steinbüchel, 1999; Solaiman et al., 2000; Steinbüchel \& Hein, 2001). Moreover, in vivo and in vitro metabolic engineering has been extensively pursued to establish new and improved PHA biosynthesis pathways and to synthesize as many different PHAs as possible from simple carbon sources provided by agriculture or from $\mathrm{CO}_{2}$ (Madison \& Huisman, 1999; Füchtenbusch et al., 2000; Steinbüchel, 2001). Only with a thorough understanding of the processes involved in the biosynthesis of PHAs will they become sufficiently cheap for bulk applications (Choi \& Lee, 1999).

Interestingly, some PHA synthases, such as the enzyme from Ralstonia eutropha, also accept mercaptoalkylCoA thioesters, such as 3-mercaptopropionyl-CoA or 3mercaptobutyryl-CoA, as substrates, thus allowing the synthesis of polythioesters (PTE). PTEs, in which the constituents are covalently linked by thioester linkages, were only recently described and they represent a new (the eighth class) of biopolymers (Lütke-Eversloh et al., 2001a, b).

Sulfur-containing biopolymers are rare and, besides those proteins that contain methionine and cysteine, some complex polysaccharides that contain sulfate groups and the PTEs, sulfur has to the best of our knowledge so far been identified only once in PHAs, in PHAs that contain 3-hydroxy-5-thiophenoxyalkanoic acids (Takagi et al., 1999). The aim of this study was to identify and metabolically engineer bacteria that synthesize PHAs with constituents containing aliphatic side chains with thioether linkages. The study was also done to shed some light on the catabolism of sulfur-containing fatty acids with respect to PHA biosynthesis in bacteria, because the catabolism of these sulfur compounds has been scarcely investigated in prokaryotic micro-organisms (Skrede et al., 1997). Such PHAs have not been described before, and they will possibly attract much interest because they are accessible to chemical reactions allowing modifications and cross-linking of the polymer chains.

\section{METHODS}

Bacteria used in this study. The $\mathrm{PHA}^{-}$mutant $\mathrm{R}$. eutropha $\mathrm{PHB}^{-} 4$ (DSM 541) and a recombinant strain of $R$. eutropha $\mathrm{PHB}^{-} 4$ harbouring plasmid pBBR1::phaC1 and expressing the PHA synthase 1 of Pseudomonas mendocina (Hein et al., 2002), as well as Pseudomonas putida KT2440 (Worsey \& Williams, 1975), P. aeruginosa PAO1 (ATCC 15692), $P$. oleovorans (ATCC 29347) and P. mendocina (DSM 50017), were used in this study.

Cultivation of bacteria. Organisms were cultured in nutrient broth (NB) or in mineral salts medium (MSM) according to Schlegel et al. (1961). Different sodium salts of alkylthio-

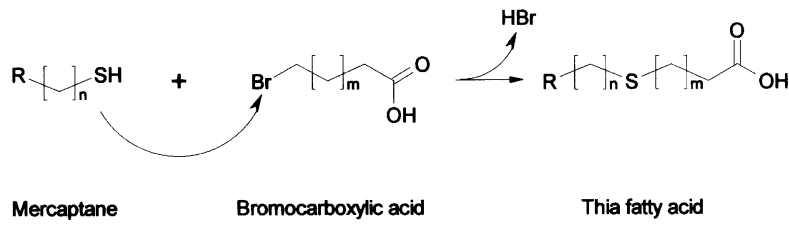

Fig. 1. Chemical synthesis of thia fatty acids.

alkanoic acids were added to these media as carbon sources, from filter-sterilized $10 \%(\mathrm{w} / \mathrm{v})$ aqueous stock solutions at the concentrations indicated in the text. Fed-batch cultures were grown under aerobic conditions at $30^{\circ} \mathrm{C}$ in 21 Erlenmeyer flasks containing $500 \mathrm{ml}$ of the appropriate medium and were agitated at 130 r.p.m. To promote PHA accumulation, the ammonia concentration was reduced to $0.05 \%(\mathrm{w} / \mathrm{v})$ in MSM.

Fed-batch cultivation of $R$. eutropha $\mathrm{PHB}^{-} 4(\mathrm{pBBR} 1:$ :phaC1) was done at a 26-litre scale in a stirred (at 50-400 r.p.m.) and aerated $\left(15-201 \mathrm{~min}^{-1}\right) 301$ stainless-steel Biostat UD30 fermenter (B. Braun, Biotech International). The fermentation was carried out in MSM. The temperature $\left(30^{\circ} \mathrm{C}\right)$ and $\mathrm{pH}$ $(7 \cdot 0)$ were automatically controlled; the airflow rate and the stirring speed were 1.0 v.v.m. [gassing vol. (culture vol. $)^{-1} \mathrm{~min}^{-1}$ ] and 400 r.p.m., respectively. Cell growth was monitored spectrophotometrically at 600 and $850 \mathrm{~nm}$. The MSM contained $1.0 \%(\mathrm{w} / \mathrm{v})$ sodium gluconate and $300 \mathrm{mg}$ kanamycin $1^{-1}$ from the beginning. Sodium gluconate and ammonium chloride were fed additionally, according to the respective growth parameters. Propylthiooctanoic acid was successively added at the end of the exponential growth phase in portions of $0.05 \%(\mathrm{w} / \mathrm{v})$ from a filter-sterilized stock solution $(10 \%, \mathrm{w} / \mathrm{v})$ at intervals of 1 or $2 \mathrm{~h}$. The final concentration of propylthiooctanoic acid (PTO) was $0.8 \%$ $(\mathrm{w} / \mathrm{v})$. At the end of the cultivation experiment, the cells were harvested by centrifugation and lyophilized.

Chemical synthesis of alkylthioalkanoic acids. Propylthioundecanoic acid (PTUD), PTO, propylthiohexanoic acid, propylthiobutyric acid, propylthiopropionic acid, butylthiovaleric acid (BTV) and octylthiohexanoic acid were chemically synthesized from alkylthiol and $\omega$-bromoalkanoic acids (Fig. 1), according to the protocol of Skrede et al. (1997). The products were identified by gas chromatography and mass spectrometry (GC/MS). Propanethiol, butanethiol, octanethiol, 11-bromoundecanoic acid, 8-bromooctanoic acid, 6-bromohexanoic acid, 5-bromovaleric acid, 4-bromobutyric acid and 3-bromopropionic acid were purchased from Sigma.

Isolation of PHAs. PHAs were extracted from lyophilized cells with hot chloroform by using a Soxhlet apparatus. The extracted crude PHAs were precipitated in 10 vols of cold methanol. To obtain highly purified polymer samples for chemical analyses, the precipitation of the polymer from a solution in chloroform was repeated at least four times. The purified polymer was finally dried under a constant stream of air.

GC/MS analysis. The polymer content and composition were determined by methanolysis of 5-7 $\mathrm{mg}$ of lyophilized cells or purified polymer in the presence of sulfuric acid; the resulting methyl esters were characterized by gas chromatography (Brandl et al., 1988).

According to the lability of thioether compounds to acidcatalysed methylation, esterification of purified polymer sam- 
Table 1. Characterization of poly(3HPTB-co-3HPTHx-co-3HPTO) obtained from different cultures of $R$. eutropha PHB-4(pBBR1::phaC1)

Cultures were grown at $30^{\circ} \mathrm{C}$ in 21 Erlenmeyer flasks or in a bioreactor, as described. The polymer yield relative to the cell dry weight was determined gravimetrically. The elemental sulfur content was analysed by the method of Grote \& Kerkeler (DIN 51768). The weight mean molar mass $\left(M_{\mathrm{W}}\right)$, number mean molar mass $\left(M_{\mathrm{N}}\right)$ and polydispersity $\left(M_{\mathrm{W}} / M_{\mathrm{N}}\right)$ of the polymers were estimated by gel-permeation chromatography relative to polystyrene standards.

\begin{tabular}{|c|c|c|c|c|}
\hline Cultivation conditions* & $\begin{array}{c}\text { Polymer content } \\
\text { (\%,w/w, of } \\
\text { CDW }+)\end{array}$ & $\begin{array}{l}\text { S content of } \\
\text { the polymer } \\
(\%)\end{array}$ & $\begin{array}{c}M_{\mathrm{W}} \\
\left(\mathrm{g} \mathrm{mol}^{-1}\right)\end{array}$ & $M_{\mathrm{W}} / M_{\mathrm{N}}$ \\
\hline Batch culture, $72 \mathrm{~h} \mathrm{NB}+0 \cdot 15 \%(\mathrm{w} / \mathrm{v})$ PTO & 43 & $16 \cdot 80$ & 105800 & $2 \cdot 3$ \\
\hline Batch culture, $72 \mathrm{~h} \mathrm{NB}+0.55 \%(\mathrm{w} / \mathrm{v})$ PTO & 43 & $17 \cdot 48$ & 110700 & $2 \cdot 5$ \\
\hline $\begin{array}{l}\text { Batch culture, } 72 \mathrm{~h} \mathrm{MSM}+1 \%(\mathrm{w} / \mathrm{v}) \text { sodium } \\
\text { gluconate }+0.55 \%(\mathrm{w} / \mathrm{v}) \text { PTO }\end{array}$ & 34 & $16 \cdot 62$ & 135000 & $2 \cdot 1$ \\
\hline $\begin{array}{l}\text { Fed-batch culture, } 45 \mathrm{~h} \mathrm{MSM}+1 \%(\mathrm{w} / \mathrm{v}) \text { sodium } \\
\text { gluconate }+16 \times 0.05 \%(\text { overall } 0.8 \%, \mathrm{w} / \mathrm{v}) \text { PTO }\end{array}$ & 24 & $14 \cdot 52$ & 102600 & $2 \cdot 4$ \\
\hline
\end{tabular}

*NB, nutrient broth; MSM, mineral salts medium.

†CDW, cell dry weight.

ples was accomplished by using (trimethylsilyl-)diazomethane as the methylating agent (Preu, 1999).

The identification of the compounds was achieved by GC/MS (HP 6890/HP 5973, Hewlett Packard).

Partial pyrolysis of polymers. Identification of the constituents of PHAs was also performed by partial pyrolysis. For this, $5 \mathrm{mg}$ of isolated polymer were transferred to the bottom of a small glass tube, which was subsequently flushed with argon and evacuated to $1 \mathrm{mbar}$. The lower part of the tube was then heated to approximately $500{ }^{\circ} \mathrm{C}$ until the polymer was completely degraded. Decomposition products condensed in the upper part of the glass tube. After cooling to room temperature, the products were dissolved in $2 \mathrm{ml}$ methanol/ chloroform $(1: 1)$. One part of this solution was used as obtained for electrospray-ionization/mass spectrometry (ESIMS), and the other part was esterified with (trimethylsilyl)diazomethane for GC/MS.

ESI-MS and ESI-MS/MS analysis. This soft ionization method was used to characterize the oligomers formed by pyrolysis of the polymers. All measurements were done on a Quattro LCZ (Micromass) apparatus with nanospray inlet.

Elemental sulfur analysis. This was performed by the Mikroanalytisches Labor Beller (Göttingen, Germany), according to the method of Grote \& Krekeler (Deutsches Institut für Normung, DIN 51768).

Molecular mass analysis. The molecular masses of the purified polyesters were estimated by gel-permeation chromatography (GPC) relative to polystyrene standards $(85,120,198,280$, $500,810$ and $990 \mathrm{kDa})$. Analysis was performed on four Styragel columns (HR 3, HR 4, HR 5, HR 6 with pore sizes of $10^{3}, 10^{4}, 10^{5}$ and $10^{6} \AA$, respectively) connected in line in a GPC apparatus (Waters). Samples were eluted with chloroform at a flow rate of $1.0 \mathrm{ml} \mathrm{min}{ }^{-1}$ and at $35^{\circ} \mathrm{C}$; the eluted compounds were monitored using a Waters 410 differential refractometer. Polydispersity and the number mean $\left(M_{N}\right)$ and weight mean $\left(M_{\mathrm{w}}\right)$ molar masses were calculated by using the Millenium Chromatography Manager GPC software (Waters).

Infrared spectroscopic analysis. The infrared spectra were taken with a fourier transform spectrometer IFS 28 (Bruker).
The samples were dissolved in $\mathrm{CHCl}_{3}$ and deposited as a film on a $\mathrm{NaCl}$ disk.

\section{RESULTS}

\section{Growth and PHA accumulation of bacteria on alkylthioalkanoic acids}

Providing fatty acids containing thioether groups, alkylthioalkanoic acids (ATAs), as carbon sources, the growth of bacteria, in which PHA biosynthesis pathways are linked to fatty acid metabolism (Steinbüchel, 2001), was investigated. To evaluate the capabilities of various pseudomonads to use ATAs as carbon and energy sources, growth experiments were carried out employing MSM agar plates which contained PTUD, PTO, octylthiohexanoic acid, BTV or propylthiopropionic acid as carbon sources. Reference growth experiments were performed on MSM agar plates which contained only sodium gluconate as sole carbon source. All Pseudomonas strains investigated in this study were able to use PTUD, PTO, octylthiohexanoic acid and BTV as sole carbon sources. Good growth, with colonies appearing after a cultivation time of $24 \mathrm{~h}$, occurred with PTUD and octylthiohexanoic acid at concentrations up to $1 \%$ $(\mathrm{w} / \mathrm{v})$ in the medium. In contrast, PTO and BTV impaired cell growth if provided at concentrations higher than $0.8 \%$ or $0.4 \%(\mathrm{w} / \mathrm{v})$, respectively. Moreover, a characteristic thiol smell was detected during cultivation of the bacteria on PTUD or BTV. Growth of a recombinant strain of $R$. eutropha $\mathrm{PHB}^{-} 4$, harbouring pBBR 1::phaC1, was very poor on any of the provided substrates. Propylthiopropionic acid was not used for growth by any of the bacteria tested.

GC analyses of cells of $P$. mendocina, $P$. aeruginos a and $P$. oleovorans did not provide evidence that the cells had synthesized PHAs containing unusual constituents after cultivation in the presence of ATAs, even if various cosubstrates were added to the medium. The medium- 

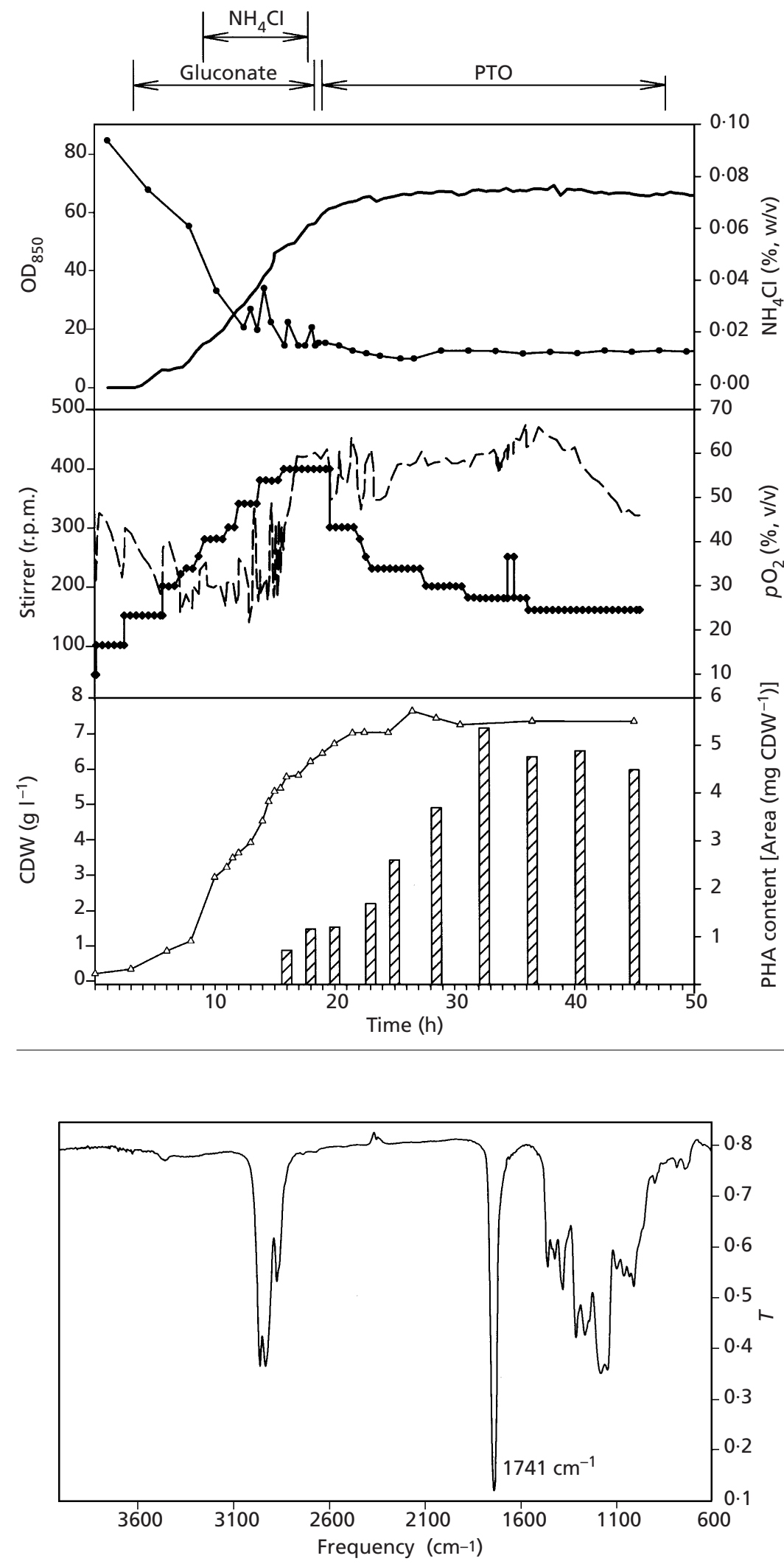

Fig. 2. Time course of a fed-batch fermentation of $R$. eutropha $\mathrm{PHB}^{-} 4$ (pBBR1::phaC1) at the 26-litre scale. The cells were cultivated for $45 \mathrm{~h}$ in MSM containing $1 \%(\mathrm{w} / \mathrm{v})$ sodium gluconate and $300 \mathrm{mg}$ kanamycin $\mathrm{I}^{-1}$. The growth curve (solid line; $\mathrm{OD}_{850}$ ), dissolved oxygen (dashed line; \% $\mathrm{pO}_{2}$ ), stirrer speed $(\bullet)$, ammonia concentration $(\bullet)$, cell dry weight $(\triangle ; C D W)$ and polymer content of the cells (hatched bars) are shown. During cultivation, $\mathrm{NH}_{4} \mathrm{Cl}$ and gluconate were fed, as indicated by the arrows. Whenever the concentration of $\mathrm{NH}_{4} \mathrm{Cl}$ was lower than $0.05 \%(w / v), 0.02 \%(w / v) \mathrm{NH}_{4} \mathrm{Cl}$ was added. If the dissolved carbon dioxide ( $\% p \mathrm{CO}_{2}$ ) decreased, gluconate was provided to the medium at concentrations of $0.2 \%(\mathrm{w} / \mathrm{v})$. PTO was added at the end of the exponential growth phase successively to a final concentration of $0.8 \%(\mathrm{w} / \mathrm{v})$. The amount of the polymer is given as 'Area (mg $(\mathrm{CDW})^{-1}$, to describe the polymer content. GC analysis did not allow quantification of the monomers, due to the lack of homogeneous reference compounds; therefore, the area as calculated by the gas chromatograph is provided.
Fig. 3. Infrared spectrum of purified poly(3HPTB-co-3HPTHx-co-3HPTO) versus $\mathrm{cm}^{-1}$, obtained from a film of the polymer on an $\mathrm{NaCl}$ disk. Peaks represent: 2983 medium $(\mathrm{CH}$, $\mathrm{CH} 2, \mathrm{CH} 3) ; 2933$ medium $(\mathrm{CH}, \mathrm{CH} 2, \mathrm{CH} 3) ; 1741$ strong (ester $\mathrm{C}=\mathrm{O}$ valence); 1380 medium; 1302 medium; 1185 strong (ester C-O); 1134 medium; 1101 medium; 1057 strong; 978 medium; 760 weak; 700 weak. chain-length PHA ( PHA $\left._{\mathrm{MCL}}\right)$ accumulated by these bacteria consisted mainly of 3-hydroxyhexanoic acid, 3-hydroxyheptanoic acid, 3-hydroxyoctanoic acid, 3- hydroxynonanoic acid, 3-hydroxydecanoic acid (3HD) and 3-hydroxydodecanoic acid, depending on the cultivation conditions. 

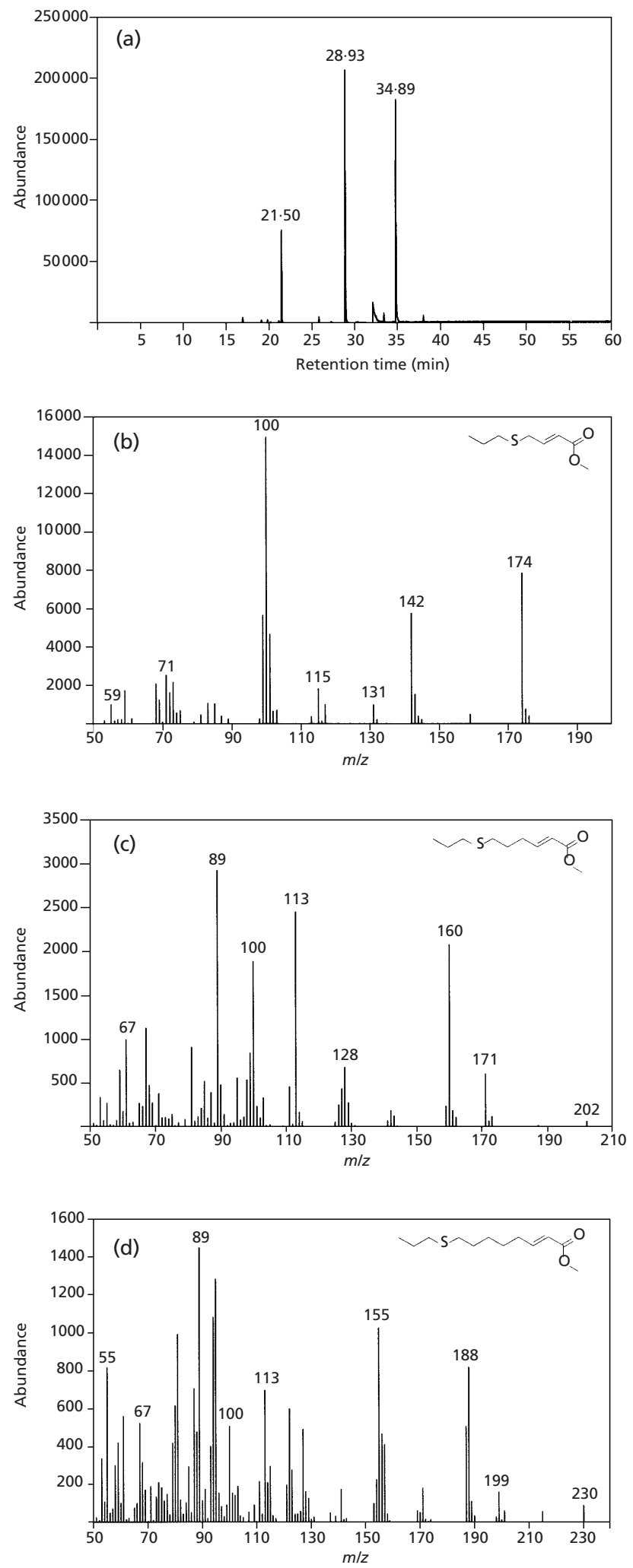

Fig. 4. GC/MS analysis of purified poly(3HPTB-co-3HPTHx-co3HPTO). The purified polymer was pyrolysed, the resulting olefins were methylated with (trimethylsilyl-)diazomethane and the methyl esters obtained were separated by GC (a). The mass spectra of the 3HPTB moiety (retention time $=21.50 \mathrm{~min}$ ) (b), the 3HPTHx moiety (retention time $=28.93 \mathrm{~min}$ ) $(\mathrm{c})$, and the

\section{PHA accumulation of $P$. putida KT2440 in the presence of PTUD}

The growth of $P$. putida KT2440 was significantly lower in comparison to the other pseudomonads when PTO or PTUD was provided as sole carbon source. Interestingly, P. putida KT2440 was the only Pseudomonas species investigated in this study for which GC analysis revealed an unusual composition of PHAs after cultivation in MSM containing $0.35 \%(\mathrm{w} / \mathrm{v})$ PTUD and equimolar amounts of nonanoic acid (v/v). Since it was suspected that the accumulated PHA contained thioether functional groups, an elemental sulfur analysis was done for the isolated polymer. It revealed a content of $6.02 \%(\mathrm{w} / \mathrm{w})$ sulfur. Besides 3-hydroxyheptanoic acid, 3-hydroxynonanoic acid, and small amounts of 3-hydroxyoctanoic acid, 3HD, 3-hydroxyundecanoic acid and 3-hydroxydodecanoic acid, a significant amount of different sulfur-containing constituents was detected by gas chromatography. However, due to the complex composition, the analyses of the partially pyrolysed polymer did not exhibit information on the exact chemical structure of these putative novel polymer constituents.

\section{Biosynthesis of poly(3-hydroxy-S-propyl- $\omega$ - thioalkanoate) [poly(3HPTA)] from PTO by a recombinant strain of $R$. eutropha}

We used a genetically engineered strain of the PHAnegative mutant $\mathrm{PHB}^{-} 4$ of $R$. eutropha harbouring plasmid pBBR1::phaC1. This strain expressed the $\mathrm{PHA}_{\mathrm{MCL}}$ synthase from $P$. mendocina, and was used for further growth and PHA-accumulation studies in which ATAs were provided as carbon sources.

Since growth of R. eutropha $\mathrm{PHB}^{-} 4$ (pBBR1::phaC1) in MSM containing PTO as sole carbon source was very poor, cultivations were carried out in complex medium (NB) or in MSM containing sodium gluconate as a second utilizable carbon source, in addition to PTO. In the presence of PTO in the medium, R. eutropha $\mathrm{PHB}^{-} 4$ (pBBR1::phaC1) synthesized a hitherto unknown polyester, revealing exclusively 3-hydroxypropylthiobutyric acid, 3-hydroxypropylthiohexanoic acid and 3-hydroxypropylthiooctanoic acid as polymer constituents [poly(3HPTB-co-3HPTHx-co-3HPTO)] (see below). The cultivation conditions and the PHA contents of the cells, as well as the sulfur contents, molecular masses and polydispersity indices of the isolated poly(3HPTA)s obtained from four different cell batches, are shown in Table 1.

To see whether larger amounts of poly(3HPTB-co3HPTHx-co-3HPTO) could be produced biotechnologically, and to obtain sufficient amounts for detailed further analysis, $R$. eutropha $\mathrm{PHB}^{-} 4(\mathrm{pBBR} 1:$ :phaC1) was cultivated at the 26 -litre scale, using a $30 \mathrm{l}$ stirred tank bioreactor. The time course of this fed-batch

3HPTO moiety (retention time $=34.89 \mathrm{~min}$ ) (d) are also presented. Due to the conjugated $\pi$-electron system, the double bond between the $\alpha$-and $\beta$-atom is the most likely structure. 


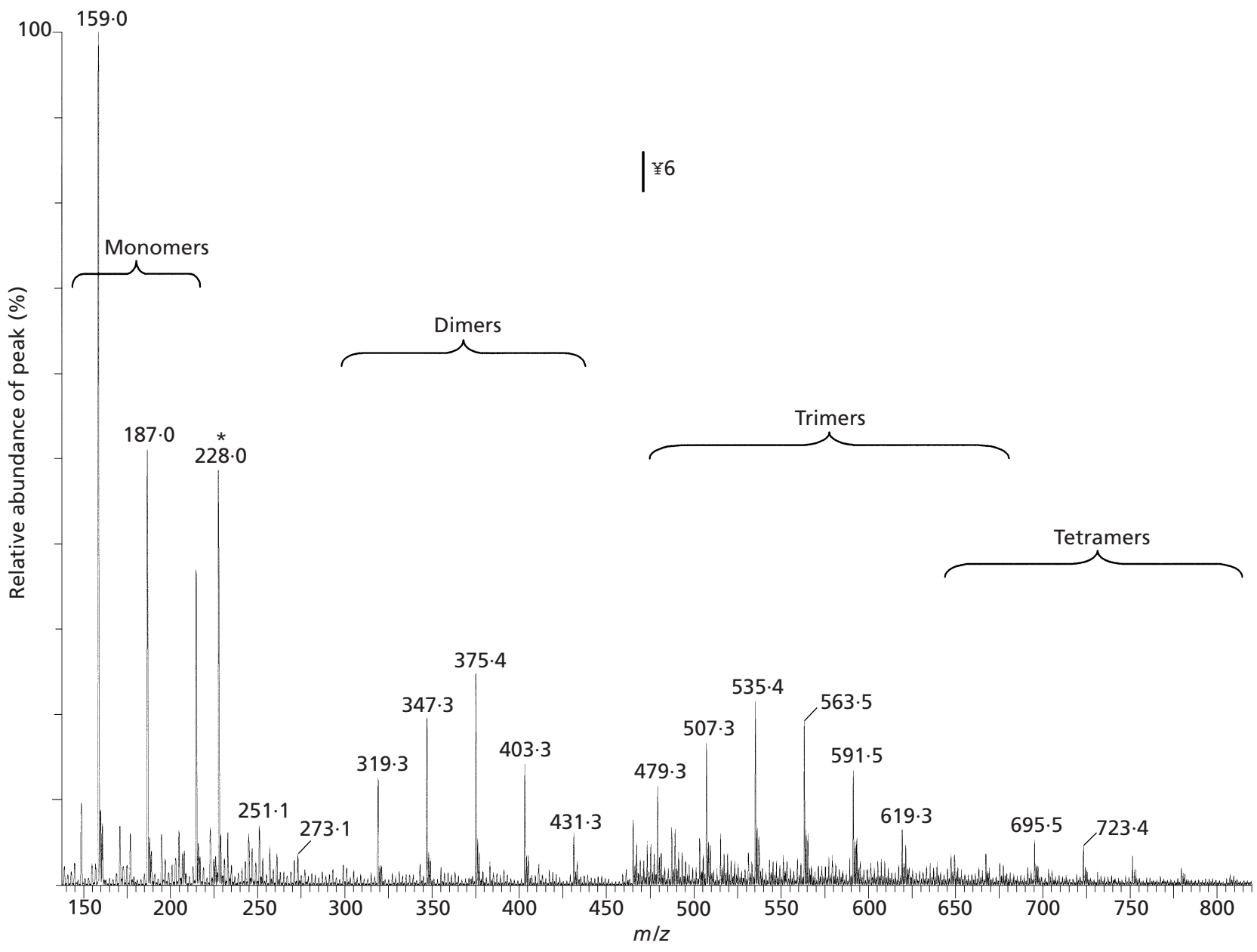

Fig. 5. Negative-ion ESI-MS analysis of partially pyrolysed poly(3HPTB-co-3HPTHx-co-3HPTO). The asterisk represents contamination. The 215 mass peak represents the olefine monomer of 3HPTO.

fermentation is presented in Fig. 2. At the end of the experiment, after $45 \mathrm{~h}$ cultivation, $190 \mathrm{~g}$ cellular dry mass was obtained and $45 \mathrm{~g}$ of the novel co-polyester could be isolated.

\section{Chemical analysis of poly(3HPTB-co-3HPTHx-co- 3HPTO)}

Infrared spectroscopic analysis of the purified polymer samples revealed a strong absorption band at $1741 \mathrm{~cm}^{-1}$, as is expected for the carbonyl vibration of an ester bond (Fig. 3), indicating polymerized constituents with carboxyl and hydroxyl functional groups. The absence of an absorption band at $1688 \mathrm{~cm}^{-1}$ clearly demonstrates that the sulfur does not occur in a thioester bond as in the previously described polythioesters (Lütke-Eversloh et al., 2001a). The presence of a thioether group cannot be clearly deduced from the infrared spectrum, because the $\mathrm{CH}_{2} \mathrm{~S}$ frequency is hidden in the complex fingerprint region.

Polymer samples were also analysed by NMR spectroscopy, indicating propyl residues. However, this analysis could not reveal the carbon chain lengths of the monomers. Moreover, functional groups in the side chains were not detectable (data not shown).

Employing the established and widely used gas chromatographic method for PHA analysis (Brandl et al., 1988), the identification of the 3HPTA methyl esters failed, because these polymer constituents were sensitive to hot acid methanolysis conditions due to the thioether groups. Therefore, other methods were chosen to analyse the polymer composition.

After pyrolysis of the polymer and subsequent methylation of the pyrolysis mixture with (trimethylsilyl-)diazomethane, three different monomeric $S$-propyl- $\omega$ thioalkenoic acids were identified by GC/MS (Fig. 4). The double bond was formed by the elimination of the adjacent fatty acid during the decomposition process. The double bond may be located either between the $\alpha$ and $\beta$-atom or between the $\beta$ - and $\gamma$-atom. Due to the conjugated $\pi$-electron system, a double bond between the $\alpha$-and $\beta$-atom is most likely. The masses of the two compounds would not differ.

The mass spectra (Fig. 4 b-d) were in good agreement with the expected structures. All spectra showed the loss 
Table 2. Some representative examples of sub-structures of poly(3HPTB-co-3HPTHx-co3HPTO), according to negative-ion ESI-MS and ESI-MS/MS analyses

\begin{tabular}{|c|c|c|}
\hline $\begin{array}{l}m / z \\
{\left[M-\mathrm{H}-\mathrm{H}_{2} \mathrm{O}\right]}\end{array}$ & $\begin{array}{c}\text { Degree of } \\
\text { polymerization }\end{array}$ & Possible sub-structures \\
\hline 159 & 1 & 3НРТВ \\
\hline 187 & 1 & 3НРТHx \\
\hline 215 & 1 & 3НРТО \\
\hline 319 & 2 & (3НРТВ-3НРТВ) \\
\hline 375 & 2 & (3НРТО-3НРТВ), (3НРТВ-3НРТО), (3НРТНx-3НРТНx) \\
\hline 535 & 3 & $\begin{array}{l}\text { (3НРТО-3НРТВ-3НРТВ), (3НРТВ-3НРТО-3НРТВ), } \\
\text { (ЗНРТВ-3НРТВ-3НРТО), (ЗНРТНx-3НРТНx-3НРТВ), } \\
\text { (3НРТНx-3НРТВ-3НРТНx), (3НРТВ-3НРТНx-3НРТНх) }\end{array}$ \\
\hline
\end{tabular}

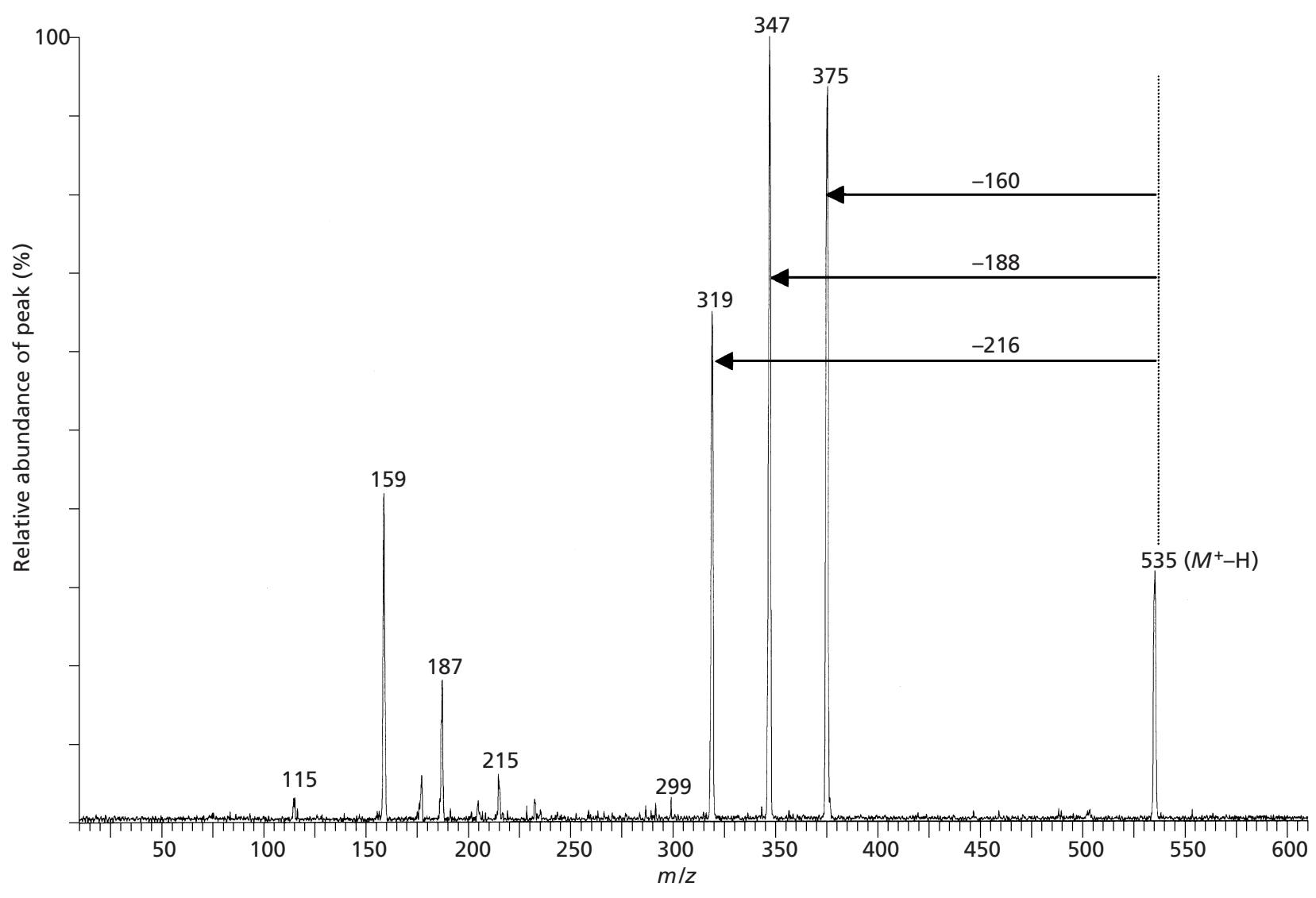

Fig. 6. Negative-ion ESI-MS/MS analysis of the 535 mass peak from partially pyrolysed poly(3HPTB-co-3HPTHx-CO-3HPTO).

of a methoxy radical and/or methanol indicating the presence of an ester group, and the loss of a propanethial and/or propylthio radical corresponding to the thiopropyl substituent. Furthermore, the presence of sulfur was clearly indicated by the characteristic isotope pattern.

Because GC analysis gave only information about the monomers, soft ionization techniques with nanospray inlet were used to identify a larger selection of substructures. Negative-ion ESI-MS (Fig. 5) showed groups of oligomers formed by combinations of 3HPTB,
3HPTHx and 3HPTO. ESI-MS/MS of the pseudomolecular ions suggested that the monomers were randomly combined (Table 2). The fragmentation of the trimer $m / z 535$ is given as an example (Fig. 6). The loss of the three possible monomers (as their corresponding olefins) with similar intensities gave proof that the trimer was a mixture of isomers with 3HPTB-, 3HPTHx- and 3HPTO-terminations.

The molar fractions of the three monomers of poly(3HPTB-co-3HPTHx-co-3HPTO) could not be determined exactly, due to the non-availability of suitable 
standards for quantification. However, mass spectrometric analyses of oligomers (see above) and calculations on the basis of data from elemental sulfur analyses indicated nearly equimolar amounts of $3 \mathrm{HPTB}$, 3HPTHx and 3HPTO.

\section{Biosynthesis of poly(3HPTA) from propylthiohexanoic acid by the recombinant strain of R. eutropha}

The recombinant mutant $\mathrm{PHB}^{-} 4$ of $\mathrm{R}$. eutropha, expressing the $P$. mendocina PHA synthase, was also cultivated on propylthiohexanoic acid under the same conditions as described above for the cultivations on PTO. The accumulated polymer was isolated and subjected to chemical analysis as described above. Infrared and NMR spectroscopy, and GC and GC/MS analysis, as well as pyrolysis of the purified polymer, revealed that the polyester consisted of 3 HPTB and 3HPTHx as sole constituents. Upon repeating similar cultivation experiments three times with propylthiobutyric acid, the cells did not accumulate any polyester that could be detected or isolated.

\section{DISCUSSION}

Using a genetically engineered bacterium, biosynthesis of novel sulfur-containing PHAs was achieved. These novel PHAs consisted of two or three different 3hydroxy-S-propyl- $\omega$-thioalkanoic acids, which have so far not been described as constituents of PHAs. The structural formulae of poly(3HPTB-co-3HPTHx-co3HPTO) and poly(3HPTB-co-3HPTHx) are shown in Fig. $7(a, b)$. The occurrence of thioether groups in the side chains provides new possibilities for chemical modifications, e.g. oxidation of the sulfur atom with iodide to form sulfonated side chains. After such modification, cross-linking of the PHA molecules will be possible. The derivatives may exhibit quite different properties after chemical modification and may thus allow special and novel technical applications, e.g. in medicine and/or pharmacy. They might be used, for example, as a skin substitute because these new polymers may have antifungal and antibacterial properties. Little is known about the physical properties of these polymers. The newly isolated polymers generally appear light yellow and translucent. Polyhydroxyalkanoates with thioether linkages in the side chain are very glutinous and exhibit a weak, but characteristic, thiol smell. They cannot be pressed into films.

As a prerequisite for growth and PHA biosynthesis, the provided carbon source(s) must be transported into the cells and subsequently metabolized to central intermediates for synthesis of other cell constituents and generation of energy, or metabolized to hydroxyacyl coenzyme A thioesters for PHA formation by PHA synthase (Anderson \& Dawes, 1990). The ATAs which were used as carbon sources in this study are probably activated to the corresponding coenzyme A thioesters by an acyl-CoA synthetase or by a CoA transferase, and are

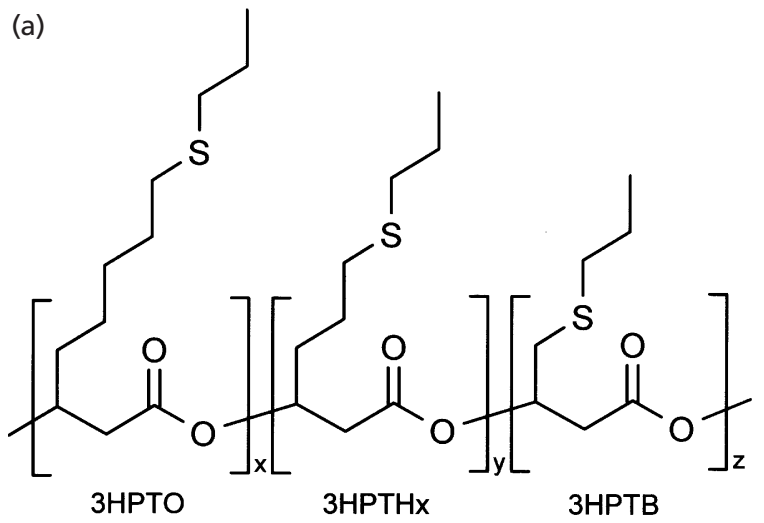

(b)

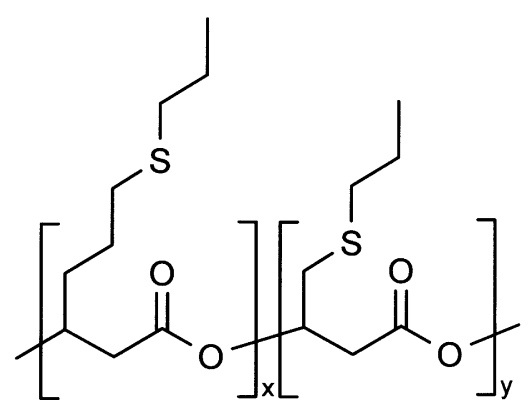

Fig. 7. Structural formulae of poly(3HPTB-co-3HPTHx-co-3HPTO) (a) and poly(3HPTB-co-3HPTHx) (b).

subsequently further catabolized via the fatty acid $\beta$-oxidation pathway. Various links between $\beta$-oxidation and PHA biosynthesis have been shown in the past (Langenbach et al., 1997; Qui et al., 1998; Fukui \& Doi, 1997; Reiser et al., 2000). Conversion of ATAs (thia fatty acids) to the corresponding CoA thioesters has been shown to occur in rat hepatocysts (Skrede et al., 1997). However, due to the thioether bonds, the derivatives cannot be completely degraded. For example, PTO is first activated to the corresponding coenzyme A thioester, and the resulting PTO-CoA thioester is then metabolized to 3HPTO-CoA, which was incorporated into PHA catalysed by the P. mendocina PHA synthase. Furthermore, the next intermediate of the $\beta$-oxidation cycle, 3-ketopropylthiooctanoic acid, is obviously cleaved by a $\beta$-ketothiolase resulting subsequently in the formation of 3HPTHx-CoA, which was also incorporated into PHAs. The next cycle of the $\beta$-oxidation then provides 3HPTB, which was also incorporated into PHAs. As a result, the terpolyester poly(3HPTB-co-3HPTHx-co-3HPTO) was synthesized and accumulated. If the cells were cultivated in the presence of propylthiohexanoic acid, the co-polyester poly(3HPTB-co-3HPTHx) was consistently synthesized. The reason why no polyester was detectable after cultivation in the presence of propylthiobutyric acid remains unknown and has yet to be elucidated.

The PHA-negative strain of $R$. eutropha $\mathrm{PHB}^{-} 4$ was not able to synthesize poly(3HPTB-co-3HPTHx-co- 


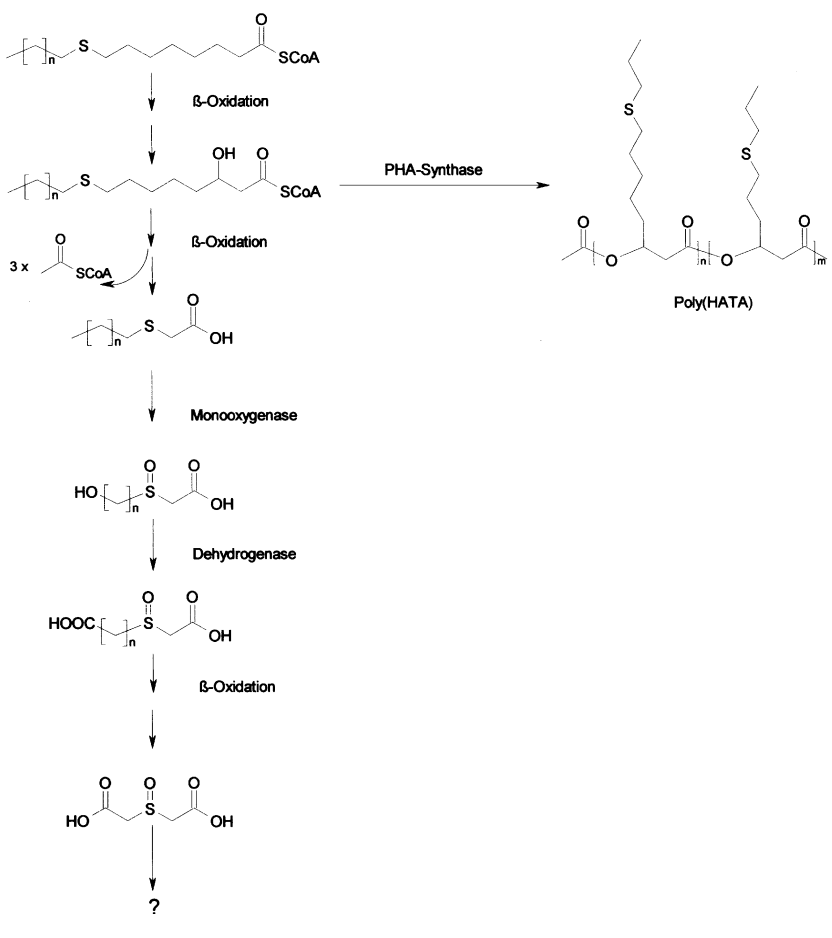

Fig. 8. Putative metabolic pathway for the biosynthesis of poly(3-hydroxyalkylthioalkanoic acid) [poly(HATA)] with an even number of carbon atoms between the thioether and the carboxyl group (adapted from Skrede et al., 1997).

3HPTO), thus confirming that a PHA synthase is involved in the synthesis of the novel PHAs. Since this strain is also not able to synthesize any other PHAs, a recombinant strain of $\mathrm{PHB}^{-} 4$ expressing the type-II PHA synthase of $P$. mendocina, which exhibited a similar structure and broad substrate range to $\mathrm{PHA}_{\mathrm{MCL}}$ synthases of other pseudomonads (Hein et al., 2002; Rehm \& Steinbüchel, 1999), was chosen as a candidate for the production of these novel PHAs. The broad substrate range of PHA synthases and in particular that of type-II PHA synthases for substrates of different carbon chain length, which also allows the incorporation of hydroxyalkanoic acids containing various substituents at the alkyl moiety, is well known (Steinbüchel \& Valentin, 1995).

Interestingly, a smell characteristic for alkylthiols occurred if pseudomonads were cultivated with ATAs that contained an odd number of carbon atoms in the carboxylic acid part of the molecule, such as PTUD or BTV. This indicates a spontaneously occurring cleavage of the 3-hydroxy-S-alkyl- $\omega$-thiopropionyl-CoA into malonic acid semialdehyde CoA and the alkylthiol, whose further metabolic fate remains to be elucidated (Skrede et al., 1997). In contrast, ATAs containing even numbers of carbon atoms in the carboxylic acid part of the molecule are presumably catabolized via the $\beta$ oxidation route to acetyl-CoA until the presence of the sulfur atom prevents another $\beta$-oxidation cycle. According to Skrede et al. (1997), the sulfur is metabolized to a sulfoxide, and further oxidation from the $\omega$-end is most likely to occur. A scheme for the putative metabolic pathway of ATA degradation and for poly(3HPTA) biosynthesis is presented in Fig. 8 .

\section{ACKNOWLEDGEMENTS}

We thank Dr Klaus Bergander (Institut für Organische Chemie, Münster) for performing NMR spectroscopic analysis and Markus Pötter (Institut für Mikrobiologie, Münster) for preparing GPC analysis.

\section{REFERENCES}

Anderson, A. J. \& Dawes, E. A. (1990). Occurrence, metabolism, metabolic role, and industrial uses of bacterial polyhydroxyalkanoates. Microbiol Rev 54, 450-472.

Brandl, H., Gross, R. A., Lenz, R. W. \& Fuller, R. C. (1988). Pseudomonas oleovorans as a source of poly( $\beta$-hydroxyalkanoates) for potential applications as biodegradable polyesters. Appl Environ Microbiol 54, 1977-1982.

Choi, J. \& Lee, S. Y. (1999). Factors affecting the economics of polyhydroxyalkanoate production by bacterial fementation. Appl Microbiol Biotechnol 51, 13-21.

Füchtenbusch, B., Wullbrandt, D. \& Steinbüchel, A. (2000). Production of polyhydroxyalkanoic acids by Ralstonia eutropha and Pseudomonas oleovorans from oil remaining from biotechnological rhamnose production. Appl Microbiol Biotechnol 53, 167-172.

Fukui, T. \& Doi, Y. (1997). Cloning and analysis of the poly(3hydroxybutyrate-co-3-hydroxyhexanoate) biosynthesis genes of Aeromonas caviae. J Bacteriol 179, 4821-4830.

Hein, S., Paletta, J. \& Steinbüchel, A. (2002). Cloning, characterization and comparison of the Pseudomonas mendocina polyhydroxyalkanoate synthases PhaC1 and PhaC2. Microbiol Biotechnol, in press.

Hocking, P. J. \& Marchessault, R. H. (1994). Biopolyesters. In Chemistry and Technology of Biodegradable Polymers, pp. 48-96. Edited by G. J. L. Griffin. London: Chapman \& Hall.

Langenbach, S., Rehm, B. H. A. \& Steinbüchel, A. (1997). Functional expression of the PHA synthase gene phaC1 from Pseudomonas aeruginosa in Escherichia coli results in poly(3hydroxyalkanoate) synthesis. FEMS Microbiol Lett 150, 303-309.

Lütke-Eversloh, T., Bergander, K., Luftmann, H. \& Steinbüchel, A. (2001a). Identification of a new class of biopolymer: bacterial synthesis of a sulfur-containing polymer with thioester linkages. Microbiology 147, 11-19.

Lütke-Eversloh, T., Bergander, K., Luftmann, H. \& Steinbüchel, A. (2001b). Biosynthesis of poly(3-hydroxybutyrate-co-3-mercaptobutyrate) as a sulfur analogue to poly(3-hydroxybutyrate) (PHB). Biomacromolecules 2, 1061-1065.

Madison, L. L. \& Huisman, G. W. (1999). Metabolic engineering of poly(3-hydroxyalkanoates): from DNA to plastic. Microbiol Mol Biol Rev 63, 21-53.

Preu, M. (1999). Experimental Design in der RückstandsanalytikOptimierung von GC/MS-Verfahren für die Analytik von Aminoglycosiden und Penicillinen. PhD thesis, Universität Aachen, Germany.

Qi, Q., Steinbüchel, A. \& Rehm, B. H. A. (1998). Metabolic routing towards polyhydroxyalkanoic acid synthesis in recombinant Escherichia coli $(\mathrm{fadR})$ : inhibition of fatty acid $\beta$-oxidation by acrylic acid. FEMS Microbiol Lett 167, 89-94. 
Rehm, B. H. A. \& Steinbüchel, A. (1999). Biochemical and genetic analysis of PHA synthases and other proteins required for PHA synthesis. Int J Biol Macromol 25, 3-19.

Reiser, S. E., Mitsky, T. A. \& Gruys, K. J. (2000). Characterization and cloning of an (R)-specific trans-2,3-enoylacyl-CoA hydratase from Rhodospirillum rubrum and use of this enzyme for PHA production in Escherichia coli. Appl Microbiol Biotechnol 53, 209-218.

Schlegel, H. G., Kaltwasser, H. \& Gottschalk, G. (1961). Ein submersverfahren zur kultur wasserstoffoxidierender bakterien: wachstumsphysologische untersuchungen. Arch Mikrobiol 38, 209-222.

Skrede, S., Sørensen, H. N., Larsen, L. N., Steineger, H. H., Høvik K., Spydevold, Ø. S., Horn, R. \& Bremer, J. (1997). Thia fatty acids, metabolism and metabolic effects. Biochim Biophys Acta 1344, $115-131$.

Solaiman, D. K. Y., Ashby, R. D. \& Foglia, T. A. (2000). Rapid and specific identification of medium-chain-length polyhydroxyalkanoate synthase gene by polymerase chain reaction. Appl Microbiol Biotechnol 53, 690-694.
Steinbüchel, A. (2001). Perspectives for biotechnological production and utilization of biopolymers: metabolic engineering of polyhydroxyalkanoate biosynthesis pathways as a successful example. Macromol Biosci 1, 1-24.

Steinbüchel, A. \& Hein, S. (2001). Biochemical and molecular basis of polyhydroxyalkanoic acids in microorganisms. Adv Biochem Eng Biotechnol 71, 81-123.

Steinbüchel, A. \& Valentin, H. E. (1995). Diversity of microbial polyhydroxyalkanoic acids. FEMS Microbiol Lett 128, 219-228.

Takagi, Y., Hashii, M., Maehara, A. \& Yamane, T. (1999). Biosynthesis of polyhydroxyalkanoate with a thiophenoxy side group obtained from Pseudomonas putida. Macromolecules 32, 8315-8318.

Worsey, M. J. \& Williams, P. A. (1975). Metabolism of toluene and xylenes by Pseudomonas putida (arvilla) $\mathrm{mt}-2$ : evidence for a new function of the TOL plasmid. J Bacteriol 124, 7-13.

Received 26 October 2001; revised 18 January 2002; accepted 22 January 2002. 